\title{
Using Virtual Reality tools to support simulations of manufacturing instances in Process Simulate: The case of an iCIM 3000 system
}

\author{
Radovan Holubek ${ }^{1, *}$, Daynier Rolando Delgado Sobrino ${ }^{1}$, Peter Košt’ál ${ }^{1}$, Roman \\ Ružarovský ${ }^{1}$ and Karol Velíšek ${ }^{1}$ \\ ${ }^{1}$ Slovak University of Technology in Bratislava, Faculty of Materials Science and Technology in \\ Trnava, Institute of Production Technologies, 91701 Trnava, Slovakia.
}

\begin{abstract}
Nowadays the trends in manufacturing are aimed at exploding and using the full potential of production devices and systems. In this context, Simulation and VR tools play a key role in the evolution of such systems specially if used in conjunction. By using these tools, the present paper focused on the analyses of an iCIM 3000 system. The main goal of the paper lied on studying the possibilities of increasing the efficiency and effectivity of whole system and its individual devices. This was achieved by means of the creation of simulations and their comparison to the real system. At the same time, the results of the simulations themselves also became the basis for further and richer analyses by means of the use of VR tools that allowed a much deeper and detailed take of the whole system. The use of VR tools offered a better assessment and comprehension of the system under study what at the same time also worked as a feedback for the improvement of the initial simulations, and this by eliminating and/or improving unnecessary movements and collisions. The results of this study have a positive significance in the improvement of the system once these are implemented.
\end{abstract}

\section{Introduction}

The current trend of increasing competition in the marketplace results in a continuous improvement of manufacturing systems and processes and thus the need of implementing and deploying or put in into practice new production ideas and designs as quickly as possible. It is precisely this fast pace, a factor that implies huge challenges and complications that are to be corrected at each stage of the production process which one way or another always affects the final commissioning time of such systems. On the other hand, extending the deployment time and debugging of new equipment also results in an increase in investment to implement these into the production process. Because of these shortcomings and complications, the use of effective methods and tools become of

* Corresponding author: radovan.holubek@stuba.sk 
tremendous importance in the reduction of the deployment times. Nowadays, most new production system projects starts from the same design tasks, i.e.: by creating CAD databases of production system models and all peripherals that are subsequently used as simulation objects in terms of designing operating production systems. This very first phase of creating accurate $\mathrm{CAD}$ models of production machines, industrial robots and other devices of a workplace is the most important stage in using such methods to design new or update/improve existing workplaces [1]. Despite a number of technology providers and system manufacturers offer their CAD databases on their devices, in many of the cases these models serve mostly as basic CAD model skeletons, often without much data for simulation (bindings, kinematic characteristics, etc.). On the other hand, it is only by using the exact CAD model of a system or process that is possible to design and properly analyze a reliable production system.

\section{Using CAD models in simulations}

Properly created CAD models can be loaded into various simulation softwares that allow to create simulations in technological and manipulation operations. The use of simulation methods in application software specifically designed for manufacturing equipment and systems are expected/designed to enable the most efficient projection of device layout solutions in terms of a proper deployment of these machines and devices. The same simulation of production and assembly processes reflects, as closely as possible, all the processes that take place in the selected case study in terms of the logistic flows of the individual equipment, the simulation of the technological and inter-operative processes, as well as the impact on the main and secondary production times.

As part of the research project "Excellent Teams of Young Researchers" - Grant STU, a project was solved with regard to the possibilities of using virtual simulation methods for the purpose of determining deviations between the virtual and the real environment. An iCIM 3000 flexible manufacturing system was chosen as a system suitable for real and virtual system comparisons; this system is located in our laboratories. The determined deviations could be first obtained in the simulation environment, then verified and better justified with Virtual Reality tools, and finally the findings and results can be implemented in the real environment/system.

First of all, it was necessary to correctly create and dismantle the CAD database of the layout according to an existing system Fig.1.

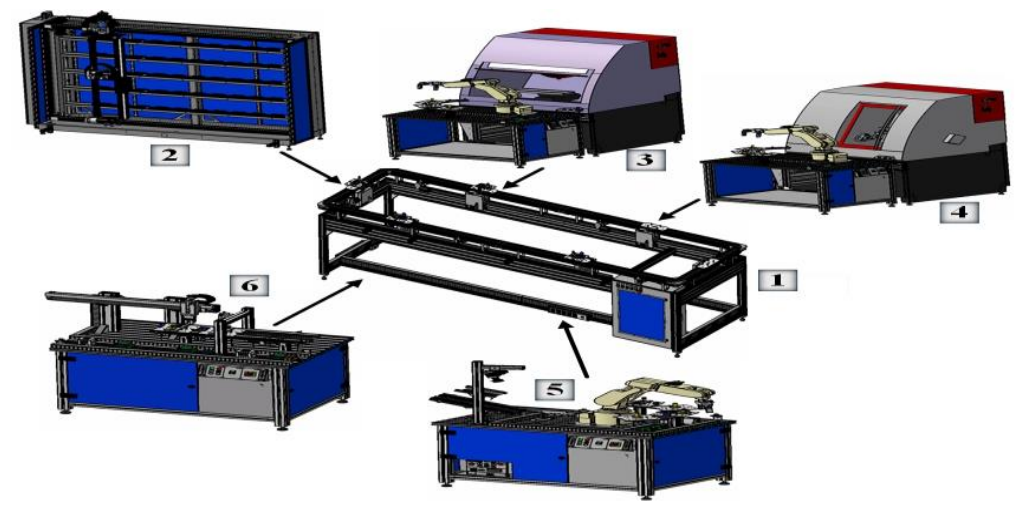

Fig. 1. CAD database of the iCIM 3000 1. Conveyor system FMF-Pallet Transfer System, 2. AS/RS Automatic Storage / Retrieval System, 3. Concept milling 105 with flexible robot feeder, 4. Concept turning 105 with flexible robot feeder, 5. FAC - Flexible Robot Assembly Cells, 6. Quality and Handling station QH 200. 
Despite the iCIM 3000 system is to be understood as a whole, for simulation needs, it was necessary to dismantle and analyze y individual stations in order to maximize the use of each station and the equipment that makes up the system.

Individual stations had to be modeled and then put together/assembled in the CATIA CAD software. These CAD models had been created as static CAD models, thus it was then necessary to convert the CAD models into ".JT files", what was done in other CAD software able to open such CATIA models and able to export them with the needed format. Such ".JT files" are able to be loaded into the Process Simulate software that is the software chosen for the iCIM3000 simulations [2]. The Process Simulate software is designed to create mechanisms with integrated CAD databases and creating off-line simulations of various production and handling operations with the possibility of further development within manufacturing systems and processes. For simulation purposes, it was necessary to create the kinematics of individual clamping devices, robbers and linear guides. This was done taking into account the exact kinematics of the RV-2SDB industrial robot and its limits on the individual axes; creating a robot's kinematics is a necessary part of a successful simulation. Besides, in Process Simulate it is was necessary to first define kinematic links, fixed and moving parts (mother and son type of relationships), TCP tools, and last but not least to define the device type as a "robot". A robot defined as "device" can be integrated into the CAD model of the non-moving parts of the CAD system loaded into Process Simulate [3]. Based on the proper loading of the CAD model of the mounting station skeleton, the insertion of the gripper and robot with the properly defined kinematics Fig.2, it was possible to create individual robot trajectories with positioning.

Based on the simulation of this assembly process, it was possible to consider the optimization of individual robot paths. This mainly refers to the fact that in the real system the robot always returns to an initial position after each operation, which increases the assembly process time. This was the main purpose of the simulation of the assembly process of the iCIM assembly station. The modification of the robot's trajectories in the assembly process represents, in terms of time, considerable time savings compared to the real system.

This way, it was necessary to properly define each single device for the simulation of each single station in the iCIM 3000 system. In the creation of the simulation, the longest phase was the integration of the CAD files into usable formats of the type JT file.

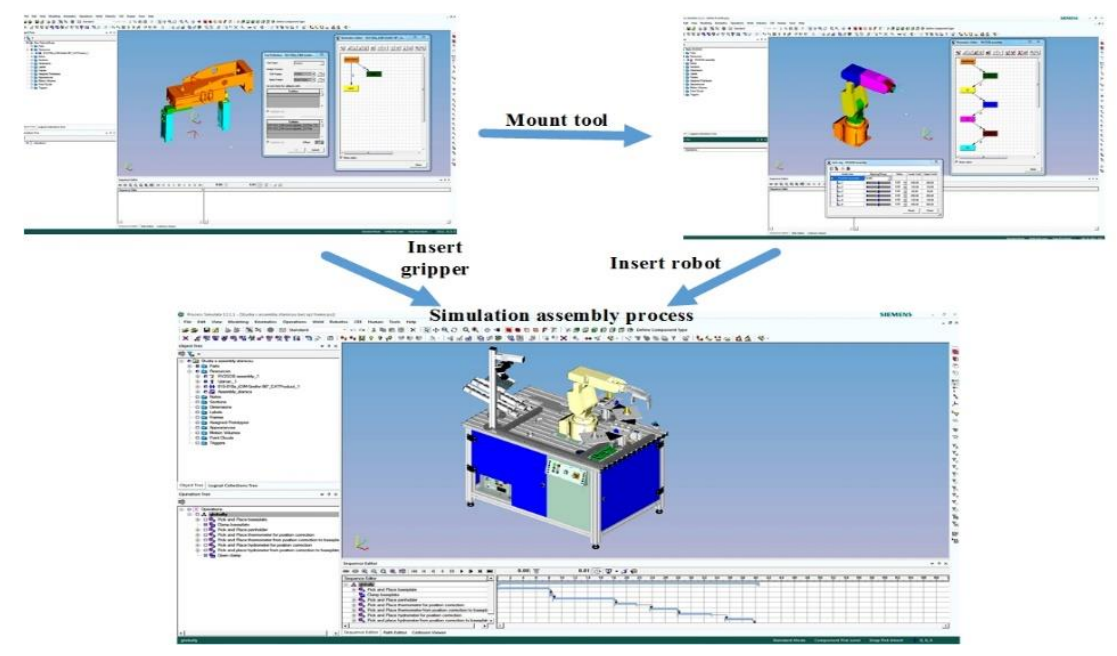

Fig. 2. Definition of device type and creation of kinematics in the assembly station. 
Kinematic linkages, definition of the type of devices and peripherals also required lots of efforts and skills [4]. This procedure had to be followed for each device, parts, and element of the assembly cell. An important aspect is that after successfully creating the iCIM3000 system database and its components, such as individual stations, robots, manipulators, grippers, clamps, conveyors and the like, it is possible to use the entire database of Process Simulate software without having to define and modify them again.

Another complicated part while creating a suitable kinematic model for simulation purposes was the storage system Fig.3. Primarily, it is a Cartesian XYZ manipulator, but the axle, in addition to the translational movement, performs rotation around its axis by $180^{\circ}$. The creation of a suitable kinematic system and linkages was preceded by the definition of the movement parts depending on the kinematic structure of the manipulator. Since this is a specific device, the creation of the links and relationship analysis was more demanding. Unfortunately this is something that is not properly covered in the manuals for Process Simulate which mainly focus on general issues. Defining the kinematic structure of the XYZ keeping in mind the sometimes simultaneous translational and rotational movements was much more challenging, this specially in terms of specifying the conditions for each part of the CAD models of the assembly. A total of 50 positions were created for the manipulator. This at the same time presupposed much more work since each position included 4 other positions for the input - output of the pallets to and from the system.

In terms of the simulation and correction of the operating time in the storage system, we were able to reduce the storage system's operating times by up to $15 \%$ based on real storage system measurements in comparison with the simulated model. This results were based on time analyzes taking into account the speed limits of the individual axes of the real storage system. This information was also further verified by Virtual Reality simulations using an HTC Vive's VR glass set. Then, the creation of the simulation scenarios of the control station with the conveyor represented the next phase of simulation in the analyses and comparison of the iCIM3000 system, see Fig.4.

The control station has the task of controlling the diameter of the holes within the tolerance range using a strain gauge sensor. In trying to integrate the control station into the simulation environment, the kinematics of the gripper (for moving the pallets), manipulator and strain gauge sensor had to be also created. The part under control is a socalled "Baseplate", which is produced on a CNC milling machine of the same iCIM 3000 real system.

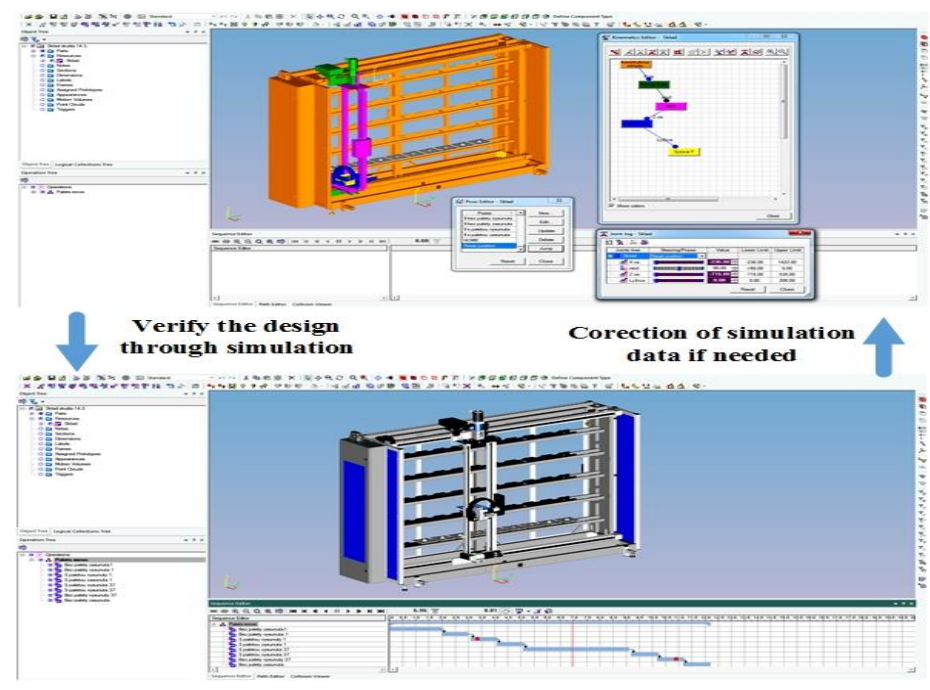

Fig. 3. Creating kinematics and positions of the storage system. 


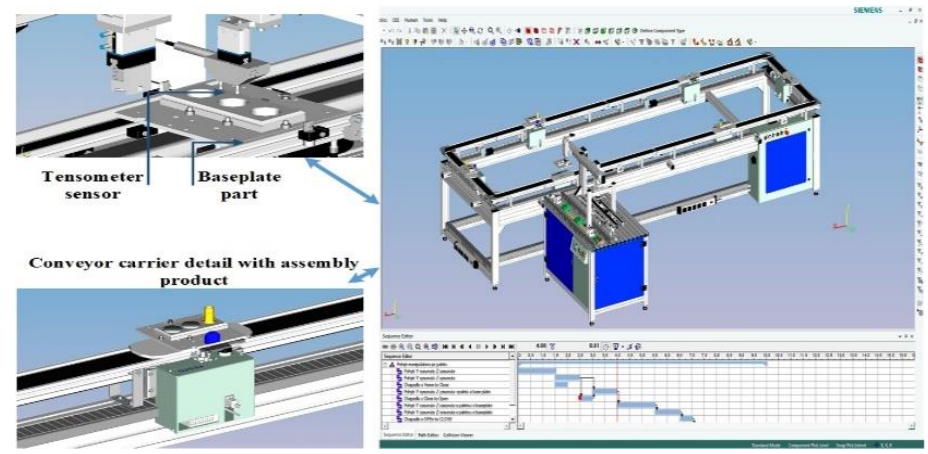

Fig. 4. Scenario of the quality control station and the conveyor.

Within the simulation scenarios, the conveyor was also integrated to connect all iCIM stations. The conveyor contains 8 pallet carriers with RFID chip identification. An important element in terms of the reducing time in comparison with the real system is that in a real system, when the conveyor stops at a station to supply a pallet, the other pallet carriers within the system stops as well till the operation, mounting for example, has been terminated. From the point of trying to reduce some of this time, this is to be eliminated by making the carriers to continue and then stop again just after the operation has been completed at a given station. This simulation verification appeared to be an appropriate elimination of the downtimes in the main and related sub-operation cycles in the comparison to the virtual and real system. This comparison of the real and simulated system with the incorporated changes was also verified by Virtual Reality simulation [5].

\section{Virtual Reality tools to support simulations}

The VR set used is the HTC Vive glasses headset. The VR includes two display panels with a resolution of $1,080 \times 1,200$ pixels. In order to create a virtual reality image, the video streams with a $90 \mathrm{~Hz}$ refresh rate. Two base station monitoring stations are used to accurately monitor the workspace and need to be placed in the corners of the room. Space monitors transmit laser beams to 32 headphones sensors and 48 sensors on handheld controllers to determine the exact position of the user in the pre-set motion space.

The tracking controls have a vibrational response that helps to intensify the virtual reality gain. The simulations we created in the Process Simulate software were used to visualize simulations in virtual environments using VR's support software. Enhancing Simulation in the Virtual Environment with HTC Vive Glasses allows for a much better visualization of the simulated process as the controls allow for $360^{\circ}$ shooting, stop simulation at any given time, hide different CAD parties that can hide possible collisions in normal simulation Fig.5.

A very important thing within the virtual environment simulation is the possibility of "breaking" the CAD parts and thus getting into hidden parts of CAD models, which can be seen in a completely different view and dimension.

The knowledge gained from the virtual environment significantly helped to eliminate major and minor times that were not originally seen in desktop PC simulation. A very important factor influencing the best visualization in CoR is the most realistic rendering in real time. In a simplified form, we can say that CAD models and / Simulation of the production process, must be able to transmit the visual experience in real time to the user without undue delay $[6,7]$. 


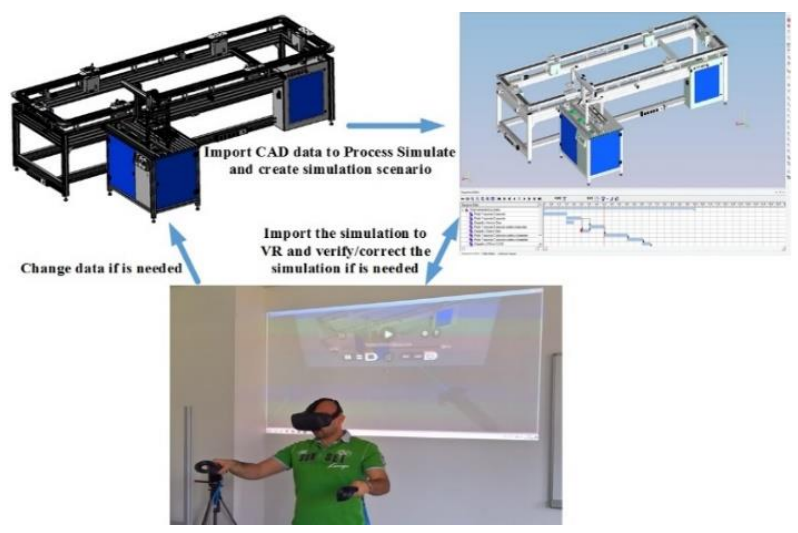

Fig. 5. Simulation scenario in virtual reality using Headset HTC Vive.

\section{Conclusions and further research}

The use of virtual reality in production systems has a huge importance in terms of safety, time-based deployment of the real system in production and the elimination of collisions, a very effective solution based on the exact verified facts within the results achieved. In order to use the VR for simulation of production systems, it is necessary to have a very good and powerful computing technique with a good graphics card. The use of VR is a very good tool because the acquired visualization is without the necessity of intervening in the real system and thus as much security as possible, especially during the incorporation of changes into the real system, increase the efficiency of comparison and overall deployment (comparison of desktop simulation, VR simulation Vs. real system), as well as the elimination of collisions, the possibility of damage to the system without prior knowledge. From the point of view of using the CoR in comparison with the real system, the use of such technologies is of great importance especially in incorporating changes in prototyping new machine and equipment products.

This research was supported by the STU Grant "Scheme for Support of Excellent Teams of Young Researchers - (1349 - Use of simulation methods in the context of the Virtual Commissioning as a tool for production systems in the Digital Factory).

\section{References}

1. K. Velíšek, R. Holubek, D.R. Delgado Sobrino, R. Ružarovský, N. Vetríková, MATEC Web of Conf, 94, online ISSN 2261-236X (2017)

2. H. Yap, Z. Taha, S. Dawal, J. Zhejiang University - Science C, 12, ISSN 1869-196X (Online) (2012)

3. L.V. Guerrero1, V. V. López, J. E. Mejía, Computer-Aided Design and Applications, 11, S11-S19, DOI: 10.1080/16864360.2014.914400 (2014)

4. M. Lorenz, M. Spranger, T. Riedel, F. Pürzel, V. Wittstock, P. Klimant, Research and Innov in Manuf: Proceedings of the 48th CIRP Conf on Manuf Sys, 41, 358-363 (2016)

5. M. Hincapi, M. Jess Ramrez, A. Valenzuela, J. Valdez, Int J for Interactive Design and Manuf, 8, 1-22, DOI: 10.1007/s12008-014-0206-7 (2014)

6. Z. Tuma, J. Tuma, R. Knoflicek, P. Blecha, F. Bradac, Procedia Engineering, 69, 1015-1020 (2014)

7. M. Eissele, O. Siemoneit, T. Ertl, IEEE Conference on, DOI: 10.1109/RAMECH.2006.252671 (2006) 